\section{LA PERCEPCIÓN DE LOS FUTUROS/AS MAESTRO/AS RESPECTO A LA IMPLANTACIÓN DE CONTENIDO DE EXPRESIÓN CORPORAL}

\author{
A PERCEPÇÃO DOS FUTUROS PROFESSORES SOBRE A \\ IMPLEMENTAÇÃO DO CONTEÚDO DA EXPRESSÃO CORPORAL C己
}

THE PERCEPTION OF FUTURE TEACHERS ABOUT IMPLEMENTATION OF TEACHING CONTENT ON BODILY EXPRESSION C己

doi' https://doi.org/10.22456/1982-8918.111735

(D)Jorge Carlos Lafuente Fernández* <gitocarlos@hotmail.com>

David Hortigüela Alcalá* <dhortiguela@ubu.es>

*Universidad de Burgos. Burgos. Espanha.
Resumen: Este artículo analiza las percepciones de los futuros maestros relativas a los problemas para impartir expresión corporal, los beneficios y los miedos de los alumnos de Primaria en esta área. La muestra fue compuesta por 18 alumnos de la asignatura de Actividades Físicas Artístico-Expresivas, de la Mención de Educación Física de la Universidad de Burgos $-23.6 \pm 5.5$ años. Se ha empleado una metodología cualitativa en la que se utilizan dos instrumentos de recogida de datos: entrevistas colectivas con los alumnos y el diario del profesor. Los resultados mostraron que tras realizar la asignatura los futuros docentes no percibían la expresión corporal como difícil de impartir, destacando la progresión de ejercicios de desinhibición, la música y el material como recursos claves. Se señala la timidez como la mayor inseguridad del alumnado, constatando cómo ésta es diferente según el género. Por último, se presenta la expresión corporal como el mejor contenido de Educación Física para desarrollar la expresión de emociones, la imaginación y la creatividad.

Palabras clave: Educación. Maestros. Percepción. Expresión Corporal.
Recibido en: 26 feb. 2021

Aprobado en: 26 may. 2021 Publicado en: 10 jun. 2021

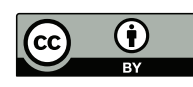

Este es un artículo publicado bajo la licencia Creative Commons Atribución 4.0 Internacional (CC BY 4.0).

eISSN: 1982-8918 


\section{INTRODUCCIÓN}

La Expresión Corporal (EC en adelante) debe estar integrada y contemplada en las acciones educativas a realizar en la escuela, debido al importante valor pedagógico como disciplina, favoreciendo el desarrollo del alumnado a nivel motriz, cognitivo, sensoperceptivo, comunicativo, expresivo y creativo (COTERÓN; SÁNCHEZ, 2010; SCHINCA, 2011). En España la presencia de la EC en la educación obligatoria aparece en la Ley General de Educación de 1970 dentro del área de Expresión Dinámica (SÁNCHEZ; LÓPEZ, 2019), más tarde la Ley Orgánica de Ordenación General del Sistema Educativo (1990) integraría definitivamente la EC como un contenido propio de la Educación Física (EF en adelante) (COTERÓN; SÁNCHEZ, 2010; MONTÁVEZ, 2012).

A pesar de su importancia en la educación y la obligatoriedad de impartir EC, marcada por la ley, esta no se trabaja en toda su amplitud desde la EF (RODRÍGUEZ et al., 2013), observándose la necesidad de afianzar la EC, como un contenido en igualdad de condiciones con respecto al resto (AIJÓN, 2012). El bloque de EC es el menos presente en las programaciones de EF, tanto en Primaria como Secundaria (MANZANO et al., 2003), siendo varias, las causas por las que ocurre esto. Por un lado, se encuentran los problemas en la formación de EC. García y Gutiérrez (2002) establecen la existencia de un gran volumen de bibliografía, sin embargo, esta no está estructurada ni unificada, encontrando un "cajón desastre" en EC integrado por multitud de contenidos. Además, Pérez de Amézaga (2015) encuentra la EC inmersa en ámbitos distintos como el pedagógico, psicológico, terapéutico, social y artístico haciendo que se afronte su estudio desde distintos ámbitos, no existiendo un acuerdo común sobre qué es la EC, cuáles son sus principios y sus contenidos (GIL, 2016).

Otro problema que se presenta, es el déficit en la formación del profesorado en EF. Así tras analizar las guías de las asignaturas de EC en el grado de Magisterio (SÁNCHEZ; LÓPEZ, 2019) y en el grado en Ciencias de la Actividad Física y el Deporte (CONESA-ROS; ANGOSTO, 2017) en las Universidades españolas, ambos trabajos concluyen, como en la mayoría de planes de estudios se presenta la EC en una única asignatura de 6 créditos, lo cual se considera escaso y residual, siendo complicado impartir todos los contenidos de forma adecuada con una carga docente insuficiente. Esto según Sánchez y López (2019) refleja una predisposición a considerar la EC como una materia de relevancia inferior en la formación del profesorado. En esta línea Robles Rodríguez et al. (2013) comentan como la EC no tiene para la mayoría de docentes de $E F$, ni prestigio ni reconocimiento, convirtiéndose en el bloque de contenidos menos valorado (ARCHILLA; PÉREZ, 2012; MACHOTA, 2007; MATANIN; COLLIER, 2003; SALINAS; VICIANA, 2006; UREÑA; ALARCÓN; UREÑA, 2009). Para evitar esto, es importante que la formación de futuros maestros, no sea solo la impartición de contenidos, sino que es necesario orientar la enseñanza a la adquisición de recursos, la construcción de la identidad profesional y el renacimiento de la vocación pedagógica (KÖPSÉN, 2014), de esta manera, cobra especial relevancia la reflexión sobre las dificultades y los beneficios de impartir EC en los futuros docentes valorando así esta, de forma realista. 
En relación al alumnado, nos encontramos con dificultades y miedos de estos en la realización de actividades expresivas. Según Robles Rodriguez et al. (2013) los alumnos a priori, manifiestan actitudes poco positivas hacia la EC, debido, según Moreno y Hellín (2007) a la forma en la que el profesor imparte los contenidos, tanto frecuencia, como metodología. Muy diferente a la que se utiliza en las prácticas deportivas.

Por otro lado, según Canales-Lacruz, Táboas-Pais y Rey-Cao (2013) la exposición a tareas expresivas es experimentada con valoraciones negativas, tales como inseguridad, timidez y vergüenza. Sintiéndose, aquel que ejecuta la actividad y es observado, como susceptible de ser juzgado por el observador (BOURDIEU, 1986), lo que podría dar lugar a bloqueos (CANALES, 2011), debido a la alta implicación emocional en EC (HANNA, 2008). Una razón por la cual en los alumnos pueden surgir sentimientos de inseguridad según Sierra (2002), es la falta de un modelo estandarizado en algunas tareas de EC. Sin embargo, Arteaga, Viciana y Conde (1999) creen que esto no es un problema, sino que podría favorecer el descubrimiento motríz individual. Los futuros docentes que realizan actividades en la asignatura de EC, tienen la oportunidad de vivenciar estas en primera persona y tras una reflexión, intuir con qué problemas podrían encontrarse también sus futuros alumnos, a la hora de realizar esas mismas tareas.

Las valoraciones negativas de los alumnos, como la timidez o la vergüenza, hacia las actividades de EC, no siempre son iguales en todos los estudiantes, existiendo una reticencia mayor dentro del género masculino (GARCÍA; GUTIÉRREZ, 2002; LLUCH et al., 2011). Además, diversos estudios indican como el alumnado femenino suele mostrar menos interés por la EF (GARZÓN et al., 2002; MIN-HAU; PHILLIPS, 2002; SALLIS; PROCHASKA; TAYLOR, 2000) fundamentalmente en relación a actividades deportivas (ARÉVALO, 2004), todo esto ha hecho que la EC se atribuya socialmente al curriculum femenino (GARCÍA; GUTIÉRREZ, 2002). Cuando los futuros docentes perciben y hacen visible este problema pueden buscar soluciones para cambiar esta dinámica.

Estas dificultades planteadas deberían ser superadas ampliamente por los beneficios que reporta a los alumnos, en relación a las capacidades cognitivomotrices (COTERÓN; SÁNCHEZ, 2010; SCHINCA, 2011) pero también por todos los beneficios a nivel afectivo, de autoestima, interpersonales o de conexión con otras áreas (LLUCH et al., 2011) y los medios que tiene la EC para crear espacios de seguridad afectivos (MEIRIEU, 1998). Además de conocer estos beneficios, es importante generar en el aula experiencias positivas en los estudiantes universitarios, según Ares y López (2012) al hablar de la formación académica en EC plantean que, cuando los contenidos impartidos se consideran relevantes para los futuros docentes, aumentan las probabilidades de ser llevados a cabo en la práctica docente.

La principal contribución del estudio es el análisis de las dificultades y los beneficios en el área de EC a partir de las percepciones de los futuros docentes. Se utiliza una metodología cualitativa a través del análisis del diario de seguimiento del profesor universitario y de las entrevistas colectivas, los cuales muestran datos que permiten comprender y reflexionar sobre las dificultades que se pueden presentar 
al impartir EC y los beneficios del trabajo de esta. El estudio atiende primero a la percepción para ver cuál es la importancia que los futuros docentes otorgan a la EC en función de sus vivencias como estudiantes en la escuela. A partir de ahí, y un vez vivenciada la asignatura en la universidad, se comprueba en qué medida esa percepción ha cambiado y cómo pretenden enseñarla cuando sean docentes en activo. Para ello se plantean tres objetivos: a) conocer los esquemas previos de los futuros docentes y la modificación de estos, tras la realización de actividades físicas artístico-expresivas, sobre las principales dificultades para impartir EC en el aula de Primaria; b) analizar las posibles inseguridades o miedos de los alumnos de Primaria, a partir de aquellos surgidos en la práctica de EC con los futuros maestros; c) reflexionar sobre los beneficios del trabajo de EC.

\section{MATERIAL Y MÉTODO}

\subsection{PARTICIPANTES}

Se utiliza una muestra por conveniencia, formada por los futuros docentes que cursaron la asignatura de Actividades Físicas Artístico-Expresivas (a partir de ahora AFAE) por la modalidad presencial y formativa, en la mención de Educación Física del Grado en Educación Primaria de la de la asignatura de Actividades Físicas Artístico-Expresivas, de la Mención Educación Física de la Universidad de Burgos, 18 sujetos (la totalidad de los inscritos en la asignatura por la vía formativa y presencial). De estos 6 chicas y 12 chicos. La media de edad fue de $23.6 \pm 5.5$ años. Todos ellos cursaron cuarto, no existiendo ningún alumno repetidor y todos tienen la experiencia de haber ejercido como maestros de prácticas el curso anterior.

La asignatura AFAE es obligatoria para los estudiantes del Grado en Educación Primaria, que deseen obtener la mención de Educación Física y fue llevada a cabo durante el primer semestre del año. Un único profesor fue el que impartió la docencia, el cual tiene una experiencia de 15 años como maestro de Primaria, mientras que fue su segundo año como responsable de esta asignatura.

\subsection{INSTRUMENTOS}

\subsubsection{Diario de seguimiento del profesor}

Tras el final de cada sesión tanto práctica como teórica, el docente de la asignatura AFAE registró los aspectos más destacados acontecidos y relativos a los ejes de estudio. Esto permitió recabar información durante todo el tiempo que se impartió la asignatura. Teniendo conocimiento de la percepción sobre cada contenido en particular. Los futuros docentes tras vivenciar la práctica en cada sesión, realizando ellos las actividades, reflexionaron sobre cómo podría influir esta, en los alumnos de Primaria. El profesor de la asignatura AFAE, anotó aquellos comentarios o comportamientos de los futuros docentes que dieran respuesta a las siguientes preguntas planteadas previamente en el diario de seguimiento del profesor (Cuadro 1). 
Cuadro 1 - Preguntas para el diario de seguimiento del profesor

1. ¿Qué comentarios han realizado los futuros docentes relacionados con la dificultad de la impartición de contenidos de EC?

2. ¿Valoran los futuros docentes los contenidos de EC?

3. ¿Destacan los futuros docentes beneficios surgidos a partir del trabajo en EC? ¿Cuáles?

4. ¿Qué actitudes se observan en los futuros docentes?

5. ¿Qué actitudes los futuros docentes destacan tras el trabajo de EC?

\subsubsection{Entrevista colectiva}

Se utilizó este instrumento para la recogida de datos, realizando una entrevista colectiva con los futuros docentes antes y al final de impartir la asignatura. Se realizó una entrevista colectiva previa, para recabar las percepciones o ideas preconcebidas que tienen los alumnos sobre la EC en general, antes de realizar la asignatura de AFAE. Y al finalizar esta, se llevó a cabo otra entrevista colectiva, para contrastar en qué modo ha cambiado esa percepción. En la entrevista colectiva se identifican elementos y hechos decisivos (FREITAS, 1999), en este caso se conversó sobre las percepciones de los problemas para impartir EC, las posibles inseguridades o miedos de los alumnos de Primaria y beneficios del trabajo de esta.

Las preguntas que se realizaron se pueden observar en Cuadro 2, estas son las mismas en la entrevista colectiva inicial y final, para poder observar como modifican sus esquemas previos los futuros docentes.

Cuadro 2 - Guion básico de la entrevista colectiva inicial y final.

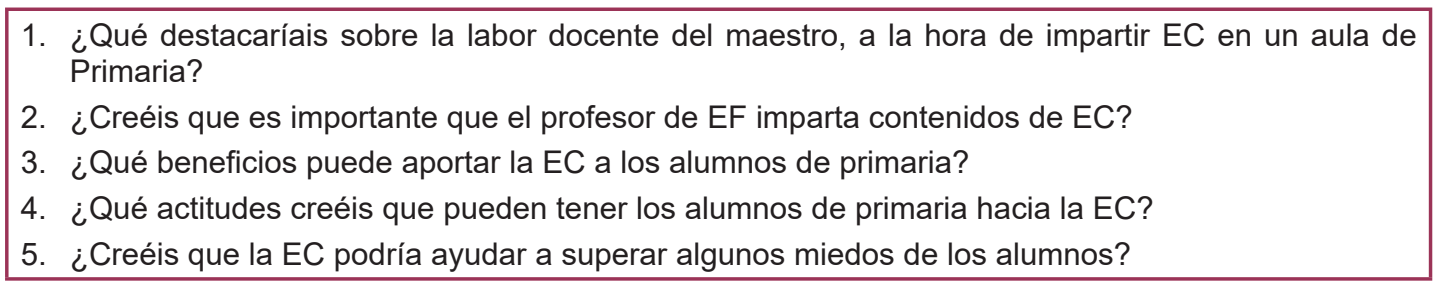

\subsection{DISEÑO Y PROCEDIMIENTO}

La investigación se llevó a cabo en tres fases.

\subsubsection{Fase 1. Delimitación y diseño del instrumento de recogida de datos}

Primero se delimitó los objetivos del estudio, la metodología a realizar y se diseñó el instrumento, las entrevistas colectivas, el diario de seguimiento del profesor, los momentos en los que se realizarían estos y cómo se llevarían a cabo. En relación a los estudiantes, debido a que el investigador impartía docencia en la asignatura AFAE, se les explicó en la primera sesión como se plantearían las entrevistas colectivas.

\subsubsection{Fase 2. Intervención, desarrollo y análisis de la información}

Se llevó a cabo la asignatura, con una duración de cuatro meses. Esta se dividió en clases teóricas y clases prácticas donde se impartieron los siguientes 
contenidos de Expresión Corporal: qué son y cómo aplicar en el aula las actividades artístico expresivas; elementos de la Expresión Corporal: comunicación, cuerpo, energía, espacio y tiempo; recursos de la EC: gesto, voz, postura, tacto y mirada; juegos de desinhibición expresivos en el aula; técnicas de expresión corporal: improvisación, juego, dramático, mimo, baile y dramatización; recursos innovadores en el aula: teatro de sombras y escenificación de situaciones deportivas. En esta fase, los futuros docentes realizaron estos contenidos vivenciándolos como alumnos. Durante este tiempo se registraron los datos y se llevó a cabo su análisis.

\subsubsection{Fase 3. Categorización de la información y análisis en profundidad de los resultados}

La información recogida de las entrevistas colectivas antes y después de impartir la asignatura fue analizada. Después se extrajeron conclusiones y se delimitaron vías de mejora para próximas actuaciones.

\subsection{ANÁLISIS EMPLEADO}

Se ha realizado una metodología de corte cualitativo, realizando el análisis de contenido a través de la revisión de la información recogida en las entrevistas colectivas a través del uso de patrones cruzados coincidentes, que favorecen la codificación y contabilización de los extractos de texto generados (SALDAÑA, 2009). Se trata de un estudio exploratorio que indaga en las percepciones de los futuros docentes de EF sobre las posibilidades educativas que tiene la expresión corporal como contenido educativo en el aula. Los resultados se organizaron en tres categorías, según un proceso de codificación abierto, axial y selectivo. Se usó la saturación como premisa previa para cribar la información, favoreciendo la contabilización de los extractos de texto y la presentación de aquellos más significativos, relacionando estos con los momentos concretos en los que han sido tomados.

\subsubsection{Generación de categorías y su categorización}

Se han generado tres categorías para estructurar los resultados:

- Dificultades para impartir la EC en el aula de Primaria: aspectos relativos a la formación docente, sus experiencias previas, el uso de material, la dificultad de los contenidos y los aspectos relacionados con recursos para ponerla en práctica en el aula.

- Posibles inseguridades o miedos de los alumnos de Primaria: diferencias de motivación entre niños y niñas, las relaciones sociales que se establecen en el aula, y aspectos emocionales relacionados con la vergüenza y la timidez.

- Beneficios del trabajo de EC: el desarrollo de la creatividad e imaginación, el uso y tipo de música empleada, las progresiones establecidas, el material utilizado y las características de las actividades puestas en práctica.

Estas categorías subyacen de la temática del estudio y de los objetivos de la investigación. La información que se establece en cada una de las categorías 
se vincula directamente con las preguntas establecidas en los dos instrumentos de recogida de datos empleados, garantizando de este modo la objetividad y linealidad de la investigación (OLIVER-HOYO; ALLEN, 2006) Además, cada categoría recoge información que atiende a diversas temáticas, las cuales han sido estructuradas en subtemas dentro de los resultados. Esta estructuración favorece la triangulación y la saturación de los datos obtenidos (TORTORELLA; VIANA; FETTERMANN, 2015). Con el fin de evitar el sesgo y garantizar la independencia en la asignación de los resultados a las categorías, se realizó un análisis independiente de cada uno de los investigadores, realizando aportaciones que posteriormente fueron consensuadas para su consignación final.

\section{RESULTADOS}

A continuación, se presenta toda la información recabada en los instrumentos de recogida de datos, agrupándose estos en las categorías planteadas en el estudio.

\subsection{DIFICULTADES PARA IMPARTIR LA EC EN EL AULA DE PRIMARIA}

En la entrevista colectiva inicial, realizada previamente a que los futuros maestros comiencen con la asignatura AFAE. Estos perciben la EC como difícil y complicada a la hora de impartir esta en un aula de Primaria. Se destaca como un contenido muy diferente al habitual en las clases de EF, lo que podría implicar el trabajo de diferentes recursos por parte de los maestros a los que no se está muy acostumbrado. Para muchos esto puede hacer que no se trabaje muy a menudo:

\footnotetext{
Considero en concreto esta disciplina de la EF una de las más difíciles de dirigir y llevar". "Va en contra de lo que mucha gente tiene asimilado como concepto de EF". "Puede resultar algo más complicado de trabajar ya que es algo diferente y que no se trabaja tanto". "Pienso que es difícil de impartir y que no en todos los colegios dedican tiempo a ello". "Es difícil de llevar a cabo puesto que no todo el mundo es capaz de poder llegar a expresarse con el cuerpo.
}

Uno de los aspectos claves que señalan los futuros docentes, es la formación. En este sentido se señala la falta de esta en los profesores de EF en relación a la EC. Se expone la importancia de adquirir recursos para poder llevarla a cabo correctamente y como con una buena formación, el docente podría implicar mejor al alumno.

Es complicado debido a la poca formación que tienen los profesores sobre este aspecto". "Muchos profesores de Educación Física trabajaron muy poco la Expresión Corporal durante su formación". "Si está bien formado, el profesor se implica en su totalidad en su función". "[...] siendo importante adquirir recursos para impartir esta.

En la información obtenida en el diario de seguimiento del profesor, a lo largo de la asignatura, los futuros maestros van puntualizando algunos aspectos importantes que se deben tener en cuenta. Primeramente, destacan la importancia de la progresión de ejercicios en relación a la desinhibición. Señalando como creen que cuando los alumnos de Primaria van realizando ejercicios poco a poco, donde no se vean expuestos, las posibilidades de que se cohíban y no trabajen es menor. 
La progresión de desinhibición planteada nos ayudará a paliar uno de los problemas que nos podemos encontrar, que es la inseguridad de algunos alumnos. Con esta progresión, los niños no se dan cuenta de que están actuando, lo que nos reduce la posibilidad de que alguno de ellos no quiera participar". "Una desinhibición a través de la progresión de ejercicios es fundamental para lograr una pérdida de vergüenza, al sentirse menos observado por los demás, según vayamos realizando ejercicios, el alumno va ganando confianza y se sentirá más seguro.

Otro aspecto que se destaca es el material. En general los futuros docentes ven positivo que en EC no se necesite un material difícil de obtener. Sin embargo, en algunos contenidos como el teatro de sombras, observan que es necesario instalaciones o material muy específicos y que no siempre se podrá acceder a este. Por último, destacan como recurso importante en EC, la música por su efecto desinhibidor y motivador.

En la mayoría de ejercicios de EC realizados no se requiere de material difícil de conseguir". "El teatro de sombras es un contenido novedoso y entretenido, pero en mi opinión difícil de llevar al aula, ya que necesitamos de una sala adecuada, una tela concreta y un foco potente". "Creo que la música ayuda mucho, ya que favorece la desinhibición". "La música elegida para realizar el baile también es importante, es fundamental que les motive.

Por último, en la entrevista colectiva final, tras impartir toda la asignatura, los futuros docentes en general, han cambiado su percepción considerando esta, más sencilla de lo que pensaban en un primer momento. Si bien mencionan lo importante de la formación, por lo que consideran necesario aumentar la carga lectiva de la asignatura actual que tiene 6 créditos y se imparte en un solo semestre.

\footnotetext{
Ahora que he cursado esta asignatura, considero que sí me veo capacitada para llevar a cabo una Unidad Didáctica de EC a los diferentes niveles de la etapa de Educación Primaria". "Creo que es mucho más sencillo de lo que parecía. Si bien, es necesaria una correcta formación del profesorado para poder llevarlo a cabo de manera adecuada". "En mi opción sería necesario tener más formación sobre este ámbito.
}

La gran mayoría de los futuros maestros muestran una gran disposición de poder realizar estos contenidos en su futura labor profesional, debido a la importancia de la EC. En este sentido destacan como ha sido un contenido habitualmente menospreciado en EF a pesar de la necesidad de trabajar esta, en las aulas de Primaria.

\footnotetext{
Viene menospreciado del pasado de la EF, pues viene dado por bailes y danzas coreografías". "Creo que es un contenido muy interesante al que se le da poco valor en Primaria". "La EC en el ámbito de la EF es algo que no se trabaja muy a menudo, puesto que yo cuando estudiaba no hice nada en cuanto a este tema". "Personalmente, sí que los trabajaría en mi futuro profesional.
}

\subsection{POSIBLES INSEGURIDADES O MIEDOS DE LOS ALUMNOS DE PRIMARIA}

En la entrevista colectiva inicial, los futuros docentes destacan la vergüenza y la timidez como el mayor problema que pueden tener los alumnos de Primaria a la hora de trabajar la EC. Sin embargo, a su vez, ven en las actividades expresivas un medio para superar esto, confiando en que a través de estas se aumente la confianza poco a poco en ellos. 
Les da vergüenza expresarse mediante el cuerpo". "Les ayudará a soltarse para perder la timidez". "Al principio puede darles vergüenza pero poco a poco lo irán superando y serán más abiertos y estarán más predispuestos a realizar incluso actividades de otro tipo"." Dependiendo de las personalidades de los alumnos será más o menos difícil de realizar, debido que aquellos que sean más tímidos les costará más hacer cosas en público.

Los futuros docentes creen que los alumnos de Primaria disfrutarán con estas actividades, entre las razones que dan, destacan la expresión y que son contenidos novedosos haciendo que les llame más la atención.

Considero que los alumnos lo disfrutan mucho". "Considero que la EC es divertida para los alumnos porque a través del movimiento (algo que en esta etapa les gusta mucho) pueden expresar sus sentimientos". "A los niños les puede llegar a gustar, porque es algo diferente a lo visto.

En el diario de seguimiento del profesor, los futuros maestros destacan la diferencia que puede haber en el trabajo de EC según el género, entre chicos y chicas. Esta diferencia se hace muy evidente tras el trabajo de bailes, donde comentan como han visto a las chicas más cómodas durante estas tareas y creen que esto puede reproducirse en el aula de Primaria.

Es apreciable la diferencia de actitud de los niños comparada con la de las niñas las cuales suelen estar mucho más dispuestas y motivadas con los bailes por lo que se implican más a la hora de realizar las actividades". "Aunque en principio resulte más cortante para los chicos que para las chicas, al tener prejuicios sobre los ritmos o parecer aburridos al sentirse observados A mí personalmente, me gustó mucho y me resulto cómodo.

Por último, en la entrevista colectiva final los futuros docentes, en línea con lo visto durante la asignatura, subrayan nuevamente los posibles problemas de vergüenza y como estos podrían afectar más a los niños que a las niñas. Volviendo a destacar en este sentido, los contenidos de desinhibición como los más efectivos para evitar dificultades relacionadas con la timidez.

Es probable que encontremos más dificultades en los chicos que en las chicas, puesto que se les ve más tímidos en general, aunque haya excepciones, en este tipo de actividades". "Para implantar contenidos de EC debemos pensar en todos los alumnos, también en los más tímidos, por lo que pueden resultar necesarias algunas sesiones de desinhibición.

\subsection{BENEFICIOS DEL TRABAJO DE EC}

En la entrevista colectiva inicial, además de aquellos beneficios que se presuponen propios de la EC, que vienen implícitos en la definición del área, como puede ser la expresión y la comunicación con el cuerpo, los futuros docentes destacan tres beneficios para los alumnos de Primaria. Por un lado, la posibilidad de mejorar las relaciones sociales, por otro el beneficio catalizador del trabajo con emociones, y por último aprender a conocerse mejor.

\footnotetext{
Herramienta útil para el desarrollo integro: físico y mental, favoreciéndose las relaciones sociales". "Les puede ayudar a conocerse a ellos mismos, así como explorar en sus capacidades y también a relacionarse con el resto de sus compañeros". "Creo que la EC es vital para que los alumnos aprendan a conocer su propio cuerpo y lo conecten con sus emociones". "Creo que
} 
sería muy útil para poder expresar sentimientos y emociones gracias al uso del cuerpo." "La expresión de emociones sería muy útil para liberar tensiones y algunos problemas de los niños que no afloran habitualmente.

Durante la realización de la asignatura, en el diario de seguimiento del profesor, se reflejan comentarios donde los futuros docentes destacan fundamentalmente lo entretenidas y divertidas que resultan las actividades de EC. Esto sirve de motivación para la mejora de las relaciones sociales, la cual vuelve a ser para ellos uno de los mayores beneficios de la EC.

\begin{abstract}
Son actividades entretenidas que generalmente gustan, y donde cambiar de compañeros ayuda para mejorar las relaciones entre los alumnos". "Creo que la sesión fue muy divertida y diferente a lo que uno esperaría dentro de la EF". "Es un contenido novedoso y entretenido". "Me parece una fantástica dinámica para ejecutar en mis clases, ya que es algo que ilusiona, divierte y motiva a los alumnos y creo que es muy beneficiosa para mejorar las relaciones entre ellos.
\end{abstract}

Por último, en la entrevista colectiva final, además de los beneficios mencionados anteriormente, como la mejora en las relaciones sociales y en la expresión de emociones. Los futuros docentes destacan el desarrollo de la imaginación y la creatividad que permite a los alumnos de Primaria ser ellos mismos.

\begin{abstract}
"Puede tener grandes beneficios como el control y gestión de las emociones. "Ayuda al alumnado a aprender a controlar su cuerpo, sus emociones y sus sentimientos, además de ayudarle a integrarse en el grupo". "Las actividades de EC pueden resultar muy positivas a la hora de desarrollar la imaginación y la creatividad". "Pudimos realizar dinámicas en las que teníamos que dar rienda suelta a nuestra imaginación, donde podemos ser nosotros mismos, algo que puede reproducirse con alumnos de Primaria.
\end{abstract}

\title{
4 DISCUSIÓN
}

La gran mayoría de los futuros docentes que estudian el Grado de Educación Primaria Mención de Educación Física, tras haber cursado la asignatura de Actividades Físicas Artístico-Expresivas han cambiado su visión de la EC percibiendo esta con menos dificultad para su impartición y señalando su intención de llevarla a cabo en su labor docente debido a su importancia. Destacan como los principales miedos de los alumnos de Primaria podrían ser la timidez y la vergüenza la cual es más habitual en chicos y en actividades de baile. Por último, destacan como beneficios fundamentales, las mejoras en las relaciones sociales, la expresión de emociones y la creatividad.

En relación a la primera categoría que trataba sobre las dificultades para impartir la EC en el aula de Primaria, los futuros docentes percibían como difíciles estos contenidos, antes de cursar la asignatura. Fundamentalmente porque son contenidos distintos a los habituales y esa puede ser la causa de no realizarse a menudo. En este sentido Aijón (2012) propone equiparar los contenidos de EC a los conocidos como tradicionales, así si los alumnos de escuela los perciben como propios de la $E F$, se evitaría esta distinción que permanece en el pensamiento de los docentes. Durante el transcurso de la asignatura, los futuros docentes señalan la progresión de ejercicios de desinhibición como uno de los recursos más importantes para evitar 
bloqueos en los alumnos de Primaria. Según Sierra (2002) en EC al no existir un modelo motriz, tan usual como en otras propuestas de EF, se provoca en el alumnado de Primaria nerviosismo e inseguridad. Es por esto que los futuros docentes ante un contenido desconocido para ellos como la EC, destacan la desinhibición como un recurso fundamental ya que como dice Meirieu (1998) esta proporciona espacios de seguridad que permiten procesos exploratorios. De esta manera será más sencillo impartir el resto de contenidos. Otros recursos que destacan los futuros maestros, es aquel relacionado con el material y los espacios, en general consideran que la EC no necesita de material difícil de conseguir, salvo en algunos contenidos como teatro de sombras. Por otro lado, señalan el uso de la música como motivador y desinhibidor.

Al final de la asignatura, en general los futuros docentes han cambiado su percepción con respecto a la dificultad de impartir la EC en Primaria, destacando como con una buena formación, esto puede ser más sencillo. Algunos autores como Archilla y Pérez (2012) apuntan problemas en la formación de EC, siendo en algunos casos inadecuada y en otras ocasiones escasa. En este sentido, los futuros docentes consideran que la actual asignatura con tan solo 6 créditos, resulta insuficiente. Sánchez y López (2019) señalan la dificultad de proporcionar una formación adecuada y generar interés en los estudiantes con un espacio curricular tan exiguo. Según Robles Rodríguez et al. (2013) esta carga horaria reducida en EC hace que muchos futuros profesores de EF muestren poco interés por impartir estos contenidos. Sin embargo, en nuestro estudio, a pesar de esto, los futuros docentes manifiestan ampliamente su intención de impartir EC en un aula de Primaria debido a su importancia.

En relación a las inseguridades o miedos en relación a la práctica de contenidos de EC, los futuros docentes señalan fundamentalmente dos. Por un lado, la vergüenza o timidez, esto explica en gran medida por qué los futuros maestros ven como muy relevantes las progresiones de desinhibición. Siguiendo a Canales (2011) acciones como observar y ser observado/a desencadenan en restricciones y bloqueos, disminuyendo la exploración y la creatividad motriz, por tanto, si no trabajamos la desinhibición para superar situaciones de vergüenza o timidez sería difícil poder trabajar todo lo demás.

Por otro lado, los futuros maestros constatan lo establecido por algunos autores (GARCÍA; GUTIÉRREZ, 2002; LLUCH et al., 2011) observando que la diferencia de género puede influir en la realización de contenidos de $E C$, señalando como los niños tienen más inseguridades sobre todo en contenidos relacionados con el baile. Los futuros maestros, deben ser conscientes de esta diferencia en el caso de que realmente exista, intentando acercar todos los contenidos a todos los alumno/as. Robles Rodriguez et al. (2013) al hablar de la diferencia de género en EC proponen "feminizar" contenidos deportivos y "masculinizar" los de EC, con esto se cambiaría la concepción tradicional de algunos contenidos haciéndolos propios de todos los alumnos, equilibrando posibles desmotivaciones derivadas por el género.

En cuanto a la última categoría, los futuros docentes solo reportan beneficios tras el trabajo de EC, no encontrándose ningún comentario negativo a este respecto. Entre los beneficios más destacados está la expresión de emociones. Hanna (2008) 
señala la elevada implicación emocional de la EC por encima del resto de contenidos de EF. Convirtiéndose la EC de esta manera, en un contenido clave para ayudar al desarrollo emocional del alumnado en la escuela. Por otro lado, los futuros maestros resaltan también la mejora de las relaciones sociales debido a las continuas interacciones con el resto de compañeros a nivel emocional. Esto está en línea con una de las principales fortalezas de la EC que señala Lluch et al. (2011).

Otro de los beneficios que subrayan los futuros docentes es el desarrollo de la imaginación y la creatividad. Este es un aspecto clave sobre todo en EF, ya que muchos contenidos pretenden la adquisición de la técnica más eficiente. Sin embargo, en las actividades de EC se permite a los alumnos realizar una exploración de las posibilidades motrices, favoreciendo de esta manera el descubrimiento de la individualidad motriz relegando la estandarización y la eficacia motriz (ARTEAGA; VICIANA; CONDE, 1999). Así la EC se convierte según los futuros docentes en el lugar donde los alumnos de Primaria podrán utilizar su imaginación y creatividad por encima del resto de contenido de EF. Todo esto hace que las actividades bien planteadas, sean percibidas como muy entretenidas y divertidas.

\section{CONCLUSIONES}

En relación al primer objetivo de estudio los futuros docentes al principio de la asignatura percibían la EC como difícil de impartir. Sin embargo, al final observan menos dificultad, mostrando la gran mayoría una alta disposición de impartir EC en un futuro. Se destaca en este grupo la progresión de ejercicios en desinhibición, la música y el material como aspectos clave que ayudarán en su impartición.

En cuanto al segundo objetivo los futuros docentes, en este grupo muestra, señalan la timidez o la vergüenza como el principal miedo de los alumnos de Primaria, pero ven en las actividades expresivas un medio para superar esto. Constatan la diferencia de género en las actividades de expresión, fundamentalmente en aquellas relacionadas con el baile.

Por último, respecto al tercer objetivo relacionado con los beneficios de la EC, los futuros maestros en el grupo estudiado perciben las actividades expresivas como muy divertidas, mejorando estas en gran medida las relaciones sociales. Por otro lado, destacan que la expresión de emociones y el desarrollo de la imaginación y la creatividad se desarrollan en EC mejor que en cualquier otro contenido de EF.

Se considera el artículo de interés para todos aquellos profesores de EF que quieran impartir EC, debido a que se les ofrece una visión realista de la EC con algunos de sus principales problemas, pero a su vez con algunas soluciones a estos. También es relevante para el ámbito de la EC a la hora de señalar debilidades y fortalezas que pueden servir de base para futuras investigaciones. 


\section{REFERENCIAS}

AIJÓN, Manuel Villard. La expresión corporal, un camino tortuoso. EmásF: revista digital de educación física, v. 14, p. 9-26, 2012.

ARCHILLA, María Teresa; PÉREZ, Darío. Dificultades del profesorado de E.F. con las actividades de expresión corporal en Secundaria. EmásF: Revista Digital de Educación Física, v. 14, p. 176-189, 2012. Disponible en: http://dialnet.unirioja.es/servlet/ articulo?codigo $=3859527$

ARES, Javier Gil.; LÓPEZ, Javier. Coterón. Relevancia de los contenidos de expresión corporal por parte de los alumnos de grado en Ciencias del Deporte. EmásF: revista digital de educación física, v. 14, p. 106-121, 2012.

ARÉVALO, Carles González. El deporte, una potencial herramienta formativa. Apunts. Educación física y deportes, v. 3, n. 77, p. 97-101, 2004.

ARTEAGA, Milagros; VICIANA, Virginia; CONDE, José Luis. Desarrollo de la expresividad corporal. Barcelona: Inde, 1999.

BOURDIEU, Pierre. Notas provisionales para la percepción social del cuerpo. In: WRIGHT MILLS, C. et al. Materiales de sociología crítica. Madrid: La piqueta, 1986. p. 183-194.

CANALES, Inma. La mirada y el tacto en la expresión corporal: consecuencias pedagógicas y propuesta de intervención. Saarbrücken: Académica Española, 2011.

CANALES-LACRUZ, Inma; TÁBOAS-PAIS, María Inés; REY-CAO, Ana. Deshibición, espontaneidad y codificación percibida por el alumnado de expresión corporal. Movimento, v. 19, n. 4 , p. $119-140,2013$.

CONESA-ROS, Elena.; ANGOSTO, Salvador. La expresión corporal y danza en la educación física de secundaria y bachillerato. Cuadernos de psicología del deporte, v. 17, n. 2, p. 111-120, 2017.

COTERÓN, Javier; SÁNCHEZ, Galo. Educación artística por el movimiento: la expresión corporal en educación física. Aula, Revista de Pedagogía de la Universidad de Salamanca, v. 16, p. 113-134, 2010.

FREITAS, Maria de Fátima Quintal. Grupos, Entrevistas Colectivas y Producción de Conocimiento Popular en Trabajos de Psicología Comunitaria. Psykhe, v.8, n.1, p. 189-194, 1999.

GARCÍA, Luis Miguel; GUTIÉRREZ, David. Análisis y estructuración de los contenidos de expresión corporal. Revistas de la Universidad, v. 2, p. 1-25, 2002.

GARZÓN, Palma Chillón et al. Actividad físico-deportiva en escolares adolescentes. Retos: nuevas tendencias en educación física, deporte y recreación, v. 3, p. 5-12, 2002.

GIL, Javier. Los contenidos de la Expresión Corporal en el Título de Grado en Ciencias del Deporte. 2016. Tesis (doctoral) - Facultad de Ciencias de la Actividad Física y del Deporte, Universidad Politécnica de Madrid, Madrid, 2016.

HANNA, Judith Lynne. A nonverbal language for imagining and learning: dance education in K-12 curriculum. Educational Researcher, v. 37, n. 8, p. 491- 506, 2008.

KÖPSÉN, Susanne. How vocational teachers describe their vocational teacher identity. Journal of Vocational Education \& Training, v. 66, n. 2, p. 194-211, 2014. 
LLUCH, Africa Calvo et al. Un análisis DAFO sobre expresión corporal desde la perspectiva de la educación física actual. EmásF: revista digital de educación física, n. 11, p. 20-28, 2011.

MACHOTA, Victoria Eugenia. El deporte y su valoración en el currículum de educación física escolar. In: CONGRESO INTERNACIONAL SOBRE LA ENSEÑANZA DE LA EDUCACIÓN FÍSICA Y EL DEPORTE ESCOLAR, 7., 2007. [Anais...]. Badajoz, [s.n.], 2007. v. 1 p. $227-$ 235.

MATANIN, Marcia; COLLIER, Connie. Longitudinal analysis of preservice teachers' beliefs about teaching physical education. Journal of teaching in physical education, v. 22, n. 2 , p. 153-168, 2003.

MANZANO, Jose Ignacio (coord). Currículo, deporte y actividad física en el ámbito escolar. La visión del profesorado de educación física en Andalucía. Málaga: Instituto Andaluz del Deporte, 2003.

MEIRIEU, Philippe. Frankestein educador. Barcelona: Laertes, 1998.

MIN-HAU, Chung; PHILLIPS, Dean Allen. The relationship between attitude toward physical education and leisure time exercise in high school students. Physical Educator, v. 59, n. 3 , p. 126, 2002.

MONTÁVEZ, Mar. La expresión corporal en la realidad educativa: Descripción y análisis de su enseñanza como punto de referencia para la mejora de la calidad docente en los centros públicos de educación primaria de la ciudad de Córdoba. 2012. Tesis (doctoral) Universidad de Córdoba, Córdoba, 2012.

MORENO, Juan Antonio; HELLíN, María. El interés del alumnado de educación secundaria obligatoria hacia la educación física. Revista electrónica de investigación educativa, $v$. 9, n. 2, p. 1-20, 2007. Disponible en: http://redie.uabc.mx/vol9no2/contenido-moreno.html. Acesso en: 31 maio 2021.

OLIVER-HOYO, María; ALLEN, DeeDee. The use of triangulation methods in qualitative educational research. Journal of College Science Teaching, v. 35, n.4, p. 42-47.

PÉREZ DE AMÉZAGA, Ana María. Estudio del método Schinca de Expresión Corporal en la Escuela Superior de Arte Dramático de Asturias. 2015. Tesis (doctoral) Universidad de Oviedo, Oviedo, 2015.

ROBLES RODRÍGUEZ, José et al. Factores que condicionan la presencia de la expresión corporal en la enseñanza secundaria según el profesorado de educación física. Retos.

Nuevas tendencias en Educación Física, Deporte y Recreación, n. 24, p. 171-175, 2013.

SALDAÑA, Johnny. The coding manual for qualitative researchers. Thousand Oaks, CA: Sage, 2009.

SALINAS, Francisco; VICIANA, Jesús. La planificación de los bloques de contenidos de la educación física en educación secundaria obligatoria. Revista digital Ciencia y Deporte, v. $3,2006$.

SALLIS, James F.; PROCHASKA, Judith J.; TAYLOR, Wendell C. A review of correlates of physical activity of children and adolescents. Medicine and science in sports and exercise, v. 32, n. 5, p. 963-975, 2000.

SÁNCHEZ, Galo; LÓPEZ, Marga. Análisis de los contenidos de expresión corporal impartidos en la formación inicial de los docentes de primaria. Educación XX1, v. 22, n. 1, p. 425-447, 2019. 
SCHINCA, Marta. Arte, expresión corporal y psicomotricidad. Cuadernos del Estudio Schinca, v. 1, n. 0, p. 31-36, 2011.

SIERRA, Miguel Ángel. La expresión corporal desde la perspectiva del alumnado de educación física. 2002. 250 p. Tesis (doctoral) - Universidad Complutense de Madrid, Madrid, 2002.

TORTORELLA, Guilherme; VIANA, Samanta; FETTERMANN, Diego. Learning cycles and focus groups: a complementary approach to the A3 thinking methodology. The Learning Organization, v. 22, n. 4, p. 229-240, 2015.

UREÑA, Nuria; ALARCÓN, Francisco; UREÑA, Fernando. La realidad de los deportes colectivos en la Enseñanza Secundaria: cómo planifican e intervienen los profesores de Murcia. Retos: nuevas tendencias en educación física, deporte y recreación, n. 16, p. 9-15, 2009. 
Resumo: Este artigo analisa as percepções dos futuros professores sobre os problemas para transmitir a expressão corporal, os benefícios e os medos dos alunos da escola primária nessa área. A amostra foi composta por 18 alunos da disciplina de Atividades Físicas Artístico-Expressivas, da Menção de Educação Física da Universidade de Burgos - 23,6 $\pm 5,5$ anos-. Foi utilizada uma metodologia qualitativa através de dois instrumentos de coleta de dados: entrevista coletiva com os alunos e diário do professor. Os resultados mostraram que, após a conclusão do curso, os futuros professores não perceberam a expressão corporal como difícil de ensinar, destacando a progressão dos exercícios de desinibição, a música e o material como recursos essenciais. A timidez é apontada como a maior insegurança dos alunos, verificando como ela difere conforme o gênero. Por fim, a expressão corporal é apresentada como o melhor conteúdo da Educação Física para desenvolver a expressão das emoções, imaginação e criatividade.

Palavras chave: Educação. Professores escolares. Percepção. Expressão Corporal.

Abstract: This article analyzes future teachers' perceptions about the problems to teach bodily expression as well as its benefits for primary school students in this area and their fears about it. The sample included 18 students from the Artistic-Expressive Physical Activities course focused on Physical Education at the University of Burgos $-23.6 \pm 5.5$ years. A qualitative methodology with two data collection instruments was employed: group interviews with students and teacher's diary. The results showed that after completing the course, future teachers did not perceive bodily expression as difficult to teach, stressing the progression of exercises for disinhibition, music and material as key resources. Shyness is pointed out as students' greatest insecurity factor and differs according to gender. Finally, bodily expression is presented as the best Physical Education content to develop expression of emotions, imagination and creativity.

Keywords: Education. School teachers. Perception. Bodily Expression. 


\section{LICENCIA DE USO}

Este es un artículo publicado en Open Access bajo la licencia Creative Commons Attribution 4.0 International (CC BY 4.0), que permite su uso, distribución y reproducción en cualquier medio, siempre que se cite correctamente la obra original. Más información en: http://creativecommons.org/licenses/by/4.0

\section{CONFLICTO DE INTERESES NOTAS EDITORIALES}

Los autores han declarado que no existe ningún conflicto de intereses en este trabajo.

\section{CONTRIBUCIONES DE LOS AUTORES}

Jorge Carlos Lafuente Fernández: conceptualización, análisis formal, investigación, metodología, recursos, validación, visualización, redacción borrador original, redacción-revisión y edición.

David Hortigüela Alcalá: conceptualización, metodología, recursos, validación, visualización, supervisión, redacción borrador original, redacción-revisión y edición

\section{FINANCIACIÓN}

O presente trabalho foi realizado sem o apoio de fontes financiadoras.

\section{ÉTICA DE LA INVESTIGACIÓN}

O projeto de pesquisa seguiu as orientações do Committee on Publication Ethics (COPE).

\section{CÓMO CITAR}

LAFUENTE FERNÁNDEZ, Jorge Carlos; HORTIGÜELA ALCALÁ, David. La percepción de los futuros/as maestro/as respecto a la implantación de contenido de expresión corporal. Movimento (Porto Alegre), v. 27, e27033, ene./dic. 2021. Disponible en: https://seer.ufrgs.br/Movimento/article/view/111735. Consultado en: [día] [mes abreviado]. [año]. DOI: https://doi.org/10.22456/1982-8918.111735

\section{RESPONSABILIDAD EDITORIAL}

Alex Branco Fraga*, Elisandro Schultz Wittizorecki*, lleana Wenetz*, Ivone Job*, Mauro Myskiw*, Raquel da Silveira*

*Universidade Federal do Rio Grande do Sul, Escola de Educação Física, Fisioterapia e Dança, Porto Alegre, RS, Brasil. 